\title{
Análisis de la ansiedad matemática en futuros emprendedores Analysis of math anxiety in future entrepreneurs
}

\author{
Mónica Maldonado ${ }^{1}$, Víctor Sotomayor ${ }^{2}$ \\ mmaldonado@edem.es, vsotomayor@edem.es \\ ${ }^{1}$ Departamento de Análisis Económico \\ Centro Universitario EDEM \\ Valencia, España \\ ${ }^{2}$ Departamento de Matemáticas y Estadística \\ Centro Universitario EDEM \\ Valencia, España
}

\begin{abstract}
Resumen- En los últimos años, las matemáticas están siendo aplicadas en gran cantidad de sectores laborales y ello hace que su importancia esté creciendo exponencialmente. Sin embargo, muchos estudiantes presentan ciertos niveles de ansiedad hacia las matemáticas que les dificultan no solo el estudio de dicha materia sino su aplicación a los diferentes ámbitos para las que son una herramienta fundamental. Con el objetivo de intentar reducir estas actitudes y comportamientos se han ido planteando una serie de experiencias docentes que involucran a los estudiantes universitarios de primeros cursos tanto del Grado en Administración y Dirección de Empresas para Emprendedores (ADE) como del Grado en Ingeniería y Gestión Empresarial (IGE) del Centro Universitario EDEM (Valencia). En concreto, se han implementado cursos de nivelación de matemáticas, una experiencia de Team Teaching; y por último, una actividad del tipo Aprendizaje Basado en Proyectos (ABP). En este trabajo se presentan dichas experiencias y la evaluación posterior de la ansiedad matemática a través de una encuesta anónima en la que se ha utilizado la escala de FennemaSherman junto con las subescalas de motivación y de confianza de Auzmendi, con el fin de valorar la reducción de los niveles de ansiedad después de la implementación de estas diferentes estrategias.
\end{abstract}

Palabras clave: ansiedad matemática, nivelación académica, team teaching, aprendizaje basado en proyectos.

\begin{abstract}
In the last years, mathematics has been applied in a large number of job sectors, and therefore its significance is growing exponentially. However, many students present certain levels of anxiety towards mathematics, and so it is difficult for them not only to study this subject, but also to apply it to different areas for which they are a fundamental tool. With the aim of trying to reduce these attitudes and behaviors, a series of teaching experiences have been proposed that involve university students in the first academic courses of both the Bachelor's Degree in Business Administration and Management (ADE) and the Bachelor's Degree in Engineering and Business Management (IGE) of the University Center EDEM. Concretely, math leveling courses have been implemented, a Team Teaching experience; and finally, an activity of type Project Based Learning (PBL). In this paper, we present these experiences and the math anxiety post evaluation through an anonymous survey, in which the FennemaSherman scale has been used together with the Auzmendi motivation and confidence subscales, in order to analyse the anxiety levels after implementing different strategies.
\end{abstract}

Keywords: math anxiety, academic leveling, team teaching, projectbased learning.

\section{INTRODUCCIÓN}

En los últimos años, las matemáticas están siendo aplicadas en gran cantidad de sectores laborales y ello hace que su importancia esté creciendo exponencialmente. El mercado de trabajo demanda no sólo profesionales STEM (Science, Technology, Engineering and Mathematics) sino profesionales de otras áreas de conocimiento que en un futuro se puedan incorporar a sectores y puestos de trabajo con un alto contenido tecnológico (CE, 2005). Sin embargo, el interés de los jóvenes por las ciencias y las matemáticas decrece continuamente, lo que provoca por un lado un menor acceso a estudios superiores STEM, y por otro lado actitudes negativas (dificultad y ansiedad) al tener que afrontar razonamientos complejos en otras disciplinas.

En este sentido, la Comisión Europea, en su comunicación "On a renewed EU agenda for higher education", indica que todos los estudiantes de educación superior, independientemente de la disciplina en la que están formándose, necesitan adquirir una serie de competencias trasversales relacionadas con la digitalización, las matemáticas, el pensamiento crítico o la capacidad de comprender y resolver problemas complejos que serán fundamentales en el desempeño de los trabajos del futuro (CE, 2017).

Las titulaciones universitarias de Grado relacionadas con la economía y la empresa incluyen asignaturas de matemáticas en sus planes de estudios. Sin embargo, se ha constatado un creciente deterioro en la formación matemática con la que acceden los nuevos estudiantes y que tiene como consecuencia unos peores resultados en las distintas asignaturas, no solo de matemáticas, sino de todas aquellas que requieren del razonamiento matemático para su comprensión: microeconomía, macroeconomía, matemáticas financieras, estadística, econometría etc. (Rodríguez et al., 2020). Algunos trabajos académicos han estudiado las consecuencias de los niveles de formación matemática preuniversitaria en los Grados de economía y empresa, obteniendo como resultado importantes discrepancias en los contenidos y profundidad de los niveles educativos previos (García y Pineda, 2020; Gil Fariña et al., 2014; Carrillo Fernández et al., 2012). Se proponen diferentes estrategias para coordinar y adecuar en los diferentes niveles educativos la formación matemática que requieren los futuros estudiantes de las titulaciones en economía y empresa y se señala también la importancia de dar a conocer 
a los estudiantes que quieran acceder a estas titulaciones la necesidad de unos conocimientos matemáticos profundos como herramienta para el razonamiento en economía (Carrillo Fernández et al., 2013).

En cuanto a las titulaciones en ingeniería, se ha producido de manera paulatina, y sobre todo desde la implantación del Espacio Europeo de Educación Superior, una reducción muy importante de la formación en matemáticas. Teniendo en cuenta la evolución de los sectores industriales, no es probable que vaya a ser suficiente para satisfacer completamente y de forma óptima las demandas del sector productivo y del mercado de trabajo (Rodríguez et. al., 2020). El nivel de conocimientos previos unido a las actitudes mostradas por los estudiantes de ingenierías está vinculado con los resultados obtenidos por los estudiantes de primeros cursos en las asignaturas relacionadas con el estudio de las matemáticas (Castro et al., 2020; 2019; 2018)

Debido en parte a los contenidos previos recibidos en las asignaturas de matemáticas preuniversitarias y a las estrategias docentes y de aprendizaje empleadas (CE, 2017), muchos estudiantes universitarios presentan ciertos niveles de ansiedad hacia las matemáticas que les dificultan no solo el estudio de dicha materia sino su aplicación a los diferentes ámbitos para las que son y serán una herramienta fundamental.

Diversos trabajos han analizado este fenómeno para los diferentes niveles educativos (Acevedo et al., 2020; Foley et al., 2017) con el objetivo de medir los diferentes aspectos relacionados con la ansiedad matemática. Son más escasos los estudios que tratan el tema en estudiantes universitarios (Nortes y Nortes, 2017; Delgado-Monge et al., 2020; Pérez-Tyteca et al., 2011; Rojas-Kramer et al., 2017).

La ansiedad matemática en estudiantes universitarios puede ser una de las causas de los bajos resultados obtenidos en aquellas materias en las que es necesario un conocimiento matemático. Sin embargo, el uso de diferentes estrategias pedagógicas puede ser efectivo en la reducción de estas actitudes y emociones y conseguir un aprendizaje más eficaz (Luttenberg et al., 2018).

El presente trabajo se enmarca en el contexto de las diferentes líneas de investigación expuestas anteriormente. Refleja la preocupación de los docentes universitarios por el nivel de conocimientos matemáticos previos de los estudiantes que acceden a las titulaciones de Grado en Administración y Dirección de Empresas (ADE) e Ingeniería y Gestión Empresarial (IGE) del Centro Universitario EDEM (Valencia) y plantea diferentes estrategias para reducir los niveles de ansiedad matemática, a la vez que aumentamos las competencias matemáticas de nuestros estudiantes con el objetivo de estén realmente preparados para su futuro laboral.

\section{CONTEXTO}

Desde hace ya algunos años y desde nuestra experiencia como docentes universitarios fuimos conscientes de la paulatina reducción de los niveles de conocimientos matemáticos con los que accedían los estudiantes tanto al Grado de ADE como de IGE. Los resultados académicos de las asignaturas de matemáticas eran cada vez peores y también lo eran las de aquellas asignaturas que necesitan de las herramientas matemáticas para su comprensión. La heterogeneidad de los itinerarios de bachiller constituye una dificultad adicional que condiciona el éxito de las asignaturas de matemáticas en los cursos iniciales de los grados de economía y empresa (Rodríguez et al., 2020).

A través de las encuestas de valoración de la docencia, que se realizan dos veces por cuatrimestre a los estudiantes, constatamos que sus actitudes ante cualquier asignatura con contenido matemático eran en muchos casos negativas. Comentarios del tipo "la asignatura es muy teórica" o "no entiendo los conceptos" eran habituales en los estudiantes encuestados.

Con el objetivo de intentar reducir estas actitudes y comportamientos se han ido planteando a lo largo de los cursos académicos del 2017/2018 al 2020/2021 una serie de experiencias docentes que involucran a los estudiantes universitarios de primeros cursos tanto del Grado en ADE para Emprendedores como del Grado en IGE del Centro Universitario EDEM (Valencia). En concreto, se han implementado, en primer lugar, cursos de nivelación académica de matemáticas tanto para los estudiantes que acceden a ambas titulaciones; en segundo lugar, experiencias de Team Teaching para los estudiantes del primer curso del Grado en ADE que involucra a las asignaturas de teoría económica con las de matemáticas; y, por último, una actividad conjunta para los estudiantes de ADE y de IGE de diferentes cursos del tipo Aprendizaje Basado en Proyectos (ABP).

La valoración de los resultados obtenidos para cada una de las estrategias aplicadas se ha ido obteniendo a través de las encuestas de valoración de cada una de ellas y han sido satisfactorios (muy bien valoradas por los estudiantes que participan en ellas, superando en todos los casos el 4 sobre 5). No obstante, durante el curso 2019/2020 se planteó la posibilidad de realizar una encuesta que midiera los niveles de ansiedad matemática de los estudiantes de primero para realizar posteriormente un seguimiento a lo largo de la titulación. Desgraciadamente, nos arrolló la pandemia de la COVID-19 y este proyecto quedó paralizado.

Durante este curso académico, hemos retomado la cuestión y se ha encuestado al total de los estudiantes de las dos titulaciones con el fin de poder evaluar la efectividad de las experiencias realizadas en la reducción de los niveles de ansiedad matemática.

\section{DESCRIPCIÓN}

Desde el curso 2017/2018 se empezaron a realizar diferentes actividades tendentes a resolver los problemas relacionados con las matemáticas que habíamos detectado. En dicho curso académico se ponen en marcha tanto los cursos de nivelación (denominados Foundations) como la actividad de Aprendizaje Basado en Proyectos (que llamamos Proyecto Trasversal). Estas dos actividades han ido evolucionando y mejorando como estrategia docente a lo largo de los diferentes cursos hasta llegar al planteamiento actual. La actividad de Team Teaching se pone en marcha en el curso 2019-2020 y ha continuado en el presente curso.

Los cursos de nivelación académica (Foundations) son cursos realizados justo antes de comenzar las clases y tienen como objetivo que los estudiantes afronten mejor los cursos matemáticos de primer año a través de la nivelación de contenidos (debido a los accesos desde diferentes centros o modalidades de bachiller). Experiencias de este tipo se han 
planteado en otras universidades con éxito, por lo que, nuestro planteamiento de los cursos de nivelación y la dimensión de los grupos contribuye a esperar unos buenos resultados (Cascón et al., 2018; De la Torre y Palacín, 2019; Picado-Alfaro, 2018; Portalés et al., 2017).

En nuestro caso, tanto en la titulación de ADE como de IGE, estos cursos han sido diseñados por los mismos docentes que van a impartir las asignaturas durante el curso, y también son impartidos por ellos mismos. De esta forma, no solo conseguimos que los conceptos y conocimientos sean los requeridos, sino que consideramos que reduce la posible ansiedad de los estudiantes en su primera toma de contacto con la realidad universitaria. Desde el curso 2018/2019, todos los estudiantes que acceden a primer curso de ambas titulaciones reciben estos cursos.

Otra de las actividades que también fue puesta en marcha durante el curso 2017/2018 es el Proyecto Trasversal. Se trata de la aplicación del proceso de Aprendizaje Basado en Proyectos (ABP) a través del diseño de una actividad colaborativa entre diferentes tipos de estudiantes. Consiste en el diseño de una idea y su posterior desarrollo hasta hacerla realidad por medio del prototipado.

La investigación actual de la aplicación del proceso de Aprendizaje Basado en Proyectos relacionada con la educación STEM demuestra que la realización de estos proyectos puede aumentar el interés de los alumnos, ya que estos deben implicarse en la resolución de problemas reales, trabajar en equipo y construir soluciones tangibles (Paredes, 2018; Domènech-Casal, 2018; Pérez, 2008).

La primera vez que se puso en marcha este proyecto fue dirigido a los estudiantes del Grado en IGE de $1^{\circ}, 2^{\circ}$ y $3^{\circ}$. Se formaron equipos con estudiantes de los diferentes cursos y se les planteó el reto de realizar todo el proceso de un producto, desde la idea hasta el prototipo. En este caso, la temática era libre y todo el proceso estuvo supervisado y valorado por un grupo de profesores. En el siguiente curso académico se producen cambios importantes, ya que se dirige tanto a los estudiantes de $\operatorname{ADE}$ como de $\operatorname{IGE}\left(1^{\circ}, 2^{\circ}\right.$ y $\left.3^{\circ}\right)$ y los equipos están formados por estudiantes de diferentes cursos y diferentes titulaciones. Por otro lado, la temática del reto deja de ser libre y pasa a ser propuesta por una empresa real. En este caso, es la empresa BSH Electrodomésticos España la que propone el reto.

En el tercer curso de implementación (2019/2020) los estudiantes a los que se dirige pasan a ser los de $1^{\circ}$ y $2^{\circ}$ tanto de ADE como de IGE, y la temática del reto es establecida por la empresa SP Berner, que propone a los equipos multidisciplinares el diseño de un producto para el hogar con material plástico reciclado.

Durante este curso (2020/2021), ante la gran acogida de los estudiantes, se repite el formato anterior (con los estudiantes de $1^{\circ}$ y $2^{\circ}$ de ambas titulaciones) y es en esta ocasión la empresa IBM la que propone el reto de crear y desarrollar un Chatbot.

Cabe mencionar que, en el diseño, la implementación, supervisión y evaluación de los diferentes proyectos están implicados tanto los diferentes profesores como la propia empresa que propone el reto. El proyecto termina con la exposición pública de los diferentes proyectos y con la selección de los tres mejores equipos.
La última de las actividades implementadas es la experiencia de Team Teaching que se puso en marcha en el curso 2019/2020 y que involucró en un primer momento a las asignaturas de Introducción a la Economía y Matemáticas I de $1^{\circ}$ de ADE.

Generalmente a los estudiantes de ADE les cuesta mucho entender que los conceptos que se les explican en las asignaturas de matemáticas son necesarios para comprender los conceptos puramente económicos. Tienen tendencia a archivar lo visto en cada asignatura en compartimentos estancos y no son capaces (en general) de relacionar las diferentes materias. Para un estudiante de economía es fundamental aprender que las matemáticas son una herramienta básica, son el lenguaje con el que se comunican generalmente los economistas. Como ocurre con cualquier lenguaje es necesario comprender lo que se dice para que éste sea eficaz.

Con este objetivo, se diseñó una experiencia colaborativa concreta utilizando para ello el concepto de derivada y su relación con el concepto económico de elasticidad. Para complementar la experiencia y conseguir que los estudiantes comprendieran mejor los conceptos a analizar introdujimos el software Geogebra (Hohenwarter et al., 2021) en las sesiones conjuntas. La literatura sugiere que el uso de la tecnología en la enseñanza de los conceptos matemáticos presenta multitud de beneficios (Drabekova y Švecova, 2015; Majerek, 2014).

Durante este curso 2020/2021 se han mantenido las sesiones de Introducción a la Economía y Matemáticas I y se ha ampliado la experiencia a las asignaturas también de $1^{\circ}$ de $\mathrm{ADE}$ de Microeconomía y Matemáticas I. El concepto que se ha trabajado conjuntamente en esta ocasión ha sido el de excedentes del consumidor y del productor relacionados con el cálculo integral (Caraballo et al., 2017).

La intención es seguir ampliando estas sesiones de trabajo conjunto involucrando otros conceptos matemáticoeconómicos, como la optimización restringida y las curvas de costes.

\section{Resultados}

El análisis de los resultados de las experiencias descritas en este trabajo está basado en los datos resultantes de una encuesta anónima que completaron de manera voluntaria un total de 289 estudiantes del Centro Universitario EDEM, tanto del Grado en Administración y Dirección de Empresas para Emprendedores (210 estudiantes) como del Grado en Ingeniería y Gestión Empresarial (79 estudiantes), en el curso 2020/2021. Entre dichos estudiantes, 94 eran alumnas y 195 eran alumnos, siendo 116 estudiantes de primer curso, 86 de segundo curso, 49 de tercer curso y 38 de cuarto curso.

El instrumento utilizado ha sido una combinación entre la Escala de Ansiedad de Fennema-Sherman (1976) y las subescalas de Motivación y Confianza del Cuestionario de Actitud hacia las Matemáticas de Auzmendi (1992). En total forman 18 ítems (12 ítems de Ansiedad, 3 de Motivación y 3 de Confianza), los cuales se puntuaban siguiendo una escala de Likert de 1 a 5, donde 1 es Totalmente en desacuerdo y 5 es Totalmente de acuerdo. Se ha seguido a Nortes y Nortes (2017) en la selección y diseño de la encuesta (ver Tablas 1, 2 y 3 ). 
En la Tabla 1 se muestran los ítems correspondientes a la ansiedad hacia las matemáticas. Es importante señalar que los ítems de ansiedad matemática se pueden agrupar en tres subescalas: Ansiedad hacia las Matemáticas en General (ítems A1, A2, A6, A7, A8 y A12), Ansiedad hacia la Resolución de Problemas (ítems A3, A9 y A10) y Ansiedad hacia los Exámenes (A4, A5 y A11).

Tabla 1. Cuestionario de Fennema-Sherman (1976)

\section{Ansiedad ante las matemáticas}

\begin{tabular}{|c|c|}
\hline A1 & Le tengo miedo a las matemáticas \\
\hline A2 & No me gustaría nada hacer más cursos de matemáticas \\
\hline A3 & $\begin{array}{l}\text { Normalmente me preocupo sobre si soy capaz de resolver } \\
\text { problemas de matemáticas }\end{array}$ \\
\hline A4 & Casi siempre me pongo nervioso en un examen de matemáticas \\
\hline A5 & Normalmente no estoy tranquilo en los exámenes de matemáticas \\
\hline A6 & Normalmente no estoy tranquilo en las clases de matemáticas \\
\hline A7 & Normalmente las matemáticas me ponen incómodo y nervioso \\
\hline A8 & $\begin{array}{l}\text { Las matemáticas me ponen incómodo, inquieto, irritable e } \\
\text { impaciente. }\end{array}$ \\
\hline A9 & $\begin{array}{l}\text { Me pongo malo cuando pienso en resolver problemas de } \\
\text { matemáticas }\end{array}$ \\
\hline A10 & $\begin{array}{l}\text { Cuando hago un problema de matemáticas se me queda la mente en } \\
\text { blanco y no soy capaz de pensar claramente }\end{array}$ \\
\hline A11 & Una prueba de evaluación de matemáticas me da miedo \\
\hline A12 & $\begin{array}{l}\text { Las matemáticas me hacen sentir preocupado, confundido y } \\
\text { nervioso }\end{array}$ \\
\hline
\end{tabular}

En la Tabla 2 se presentan los tres ítems que miden la motivación de los estudiantes hacia el estudio de las matemáticas. Los ítems correspondientes a la confianza que causa adquirir cierta habilidad en matemáticas se muestran en la Tabla 3.

Tabla 2. Subescala de Motivación de Auzmendi (1992) Motivación hacia las matemáticas

\begin{tabular}{|c|l|}
\hline M1 & $\begin{array}{l}\text { La matemática es demasiado teórica para que pueda servirme de } \\
\text { algo }\end{array}$ \\
\hline M2 & $\begin{array}{l}\text { Las matemáticas pueden ser útiles para el que decida realizar una } \\
\text { carrera de "ciencias", pero no para el resto de los estudiantes }\end{array}$ \\
\hline M3 & $\begin{array}{l}\text { La materia que se imparte en las clases de matemáticas es muy poco } \\
\text { interesante }\end{array}$ \\
\hline
\end{tabular}

Tabla 3. Subescala de Confianza de Auzmendi (1992)

Confianza ante las matemáticas

\begin{tabular}{c|l}
\hline C1 & $\begin{array}{l}\text { Tener buenos conocimientos de matemáticas incrementará mis } \\
\text { posibilidades de trabajo }\end{array}$ \\
\hline C2 & $\begin{array}{l}\text { Me provoca una gran satisfacción el llegar a resolver problemas de } \\
\text { matemáticas }\end{array}$ \\
\hline C3 & $\begin{array}{l}\text { Si me lo propusiera creo que llegaría a dominar bien las } \\
\text { matemáticas }\end{array}$ \\
\hline
\end{tabular}

Se ha analizado la consistencia interna de las escalas a través del coeficiente Alfa de Cronbach (Márquez, 2004). El coeficiente alfa de Cronbach de la encuesta realizada a nivel global es de 0.87 , mientras que por separado tenemos que el apartado de Ansiedad posee un coeficiente 0.92, el apartado de Motivación un 0.64 y el apartado de Confianza un 0.65.
En la Tabla 4 mostramos las medias y desviaciones típicas de los 12 ítems presentes en la encuesta sobre ansiedad matemática para el conjunto de los estudiantes encuestados, mientras que en la Tabla 5 hacemos lo mismo para los ítems de motivación y confianza. Los 12 ítems de la escala de ansiedad matemática así como los 3 ítems de confianza están expresados todos en forma positiva de forma que un resultado mayor indica un mayor nivel de ansiedad.

Tabla 4. Media y desviación típica de los ítems de Ansiedad

\begin{tabular}{ccccccc}
\hline & $\mathbf{A 1}$ & $\mathbf{A 2}$ & $\mathbf{A 3}$ & $\mathbf{A 4}$ & $\mathbf{A 5}$ & $\mathbf{A 6}$ \\
\hline Media & 2.3 & 2.3 & 3.2 & 3.0 & 2.9 & 2.2 \\
Desv. Típica & 1.15 & 1.21 & 1.17 & 1.3 & 1.26 & 1.08 \\
\hline & & & & & & \\
\hline & $\mathbf{A 7}$ & $\mathbf{A 8}$ & $\mathbf{A 9}$ & $\mathbf{A 1 0}$ & $\mathbf{A 1 1}$ & $\mathbf{A 1 2}$ \\
\hline Media & 2.2 & 2.1 & 2.2 & 2.2 & 2.7 & 2.3 \\
Desv. Típica & 1.07 & 1.07 & 1.08 & 1.07 & 1.2 & 1.09 \\
\hline
\end{tabular}

Hemos asociado a los ítems de motivación su valor complementario, pues estos están formulados en forma negativa, con el objetivo de que un mayor valor del resultado indique una mayor motivación de los estudiantes.

Tabla 5. Media y desviación típica de los ítems de Motivación y Confianza

\begin{tabular}{ccccccc}
\hline & M1 & M2 & M3 & C1 & C2 & C3 \\
\hline Media & 3.9 & 4.0 & 3.5 & 4.1 & 4.5 & 4.3 \\
Desv. Típica & 1.15 & 1.13 & 1.03 & 1.0 & 0.86 & 0.88 \\
\hline
\end{tabular}

En las Tablas 6 y 7 mostramos las medias y las desviaciones típicas obtenidas en conjunto para la titulación de ADE y de IGE, respectivamente, agrupando las diferentes subescalas. Como puede verse, el nivel general de ansiedad matemática es inferior al nivel neutral en ambas titulaciones (esto es, inferior a 3).

Tabla 6. Media y desviación típica de las subescalas por cursos de ADE

\begin{tabular}{cccccc}
\hline & & $\mathbf{1}^{\mathbf{0}}$ & $\mathbf{2}^{\mathbf{0}}$ & $\mathbf{3}^{\mathbf{0}}$ & $\mathbf{4}^{\mathbf{0}}$ \\
\hline $\begin{array}{c}\text { Ansiedad hacia } \\
\text { las Matemáticas } \\
\text { en General }\end{array}$ & Mesv. Típica & 0.91 & 0.90 & 0.77 & 0.90 \\
\hline $\begin{array}{c}\text { Ansiedad hacia } \\
\text { la Resolución de } \\
\text { Problemas }\end{array}$ & Mesv. Típica & 0.88 & 0.87 & 0.73 & 0.93 \\
\hline $\begin{array}{c}\text { Ansiedad hacia } \\
\text { los exámenes }\end{array}$ & Media & 3.1 & 2.9 & 2.6 & 2.9 \\
\hline Mesv. Típica & 1.15 & 1.15 & 0.90 & 1.03 \\
\hline Motivación & Media & 3.5 & 3.8 & 4.0 & 3.7 \\
\hline Confianza & Media & 4.3 & 4.2 & 4.2 & 4.1 \\
& Desv. Típica & 0.61 & 0.84 & 0.73 & 0.79 \\
\hline
\end{tabular}


Tabla 7. Media y desviación típica de las subescalas por cursos de IGE

\begin{tabular}{cccccc}
\hline & & $\mathbf{1}^{\mathbf{0}}$ & $\mathbf{2}^{\mathbf{0}}$ & $\mathbf{3}^{\mathbf{0}}$ & $\mathbf{4}^{\mathbf{0}}$ \\
\hline $\begin{array}{c}\text { Ansiedad hacia } \\
\text { las Matemáticas } \\
\text { en General }\end{array}$ & Media & 2.3 & 2.0 & 2.1 & 1.5 \\
Desv. Típica & 0.95 & 0.52 & 0.69 & 0.62 \\
\hline $\begin{array}{c}\text { Ansiedad hacia } \\
\text { la Resolución de } \\
\text { Problemas }\end{array}$ & Media & 2.6 & 2.1 & 2.1 & 1.5 \\
\hline $\begin{array}{c}\text { Ansiedad hacia } \\
\text { los exámenes }\end{array}$ & Media & 3.1 & 2.8 & 2.5 & 1.8 \\
\hline Mosvica Típica & 1.17 & 1.02 & 0.74 & 0.79 \\
\hline Confianza & Media & 3.9 & 3.9 & 4.1 & 4.3 \\
& Desv. Típica & 0.85 & 1.00 & 0.62 & 0.65 \\
\hline & Media & 4.6 & 4.5 & 4.6 & 4.3 \\
\hline
\end{tabular}

Finalmente, cabe mencionar que se ha estudiado también la posible correlación existente entre las escalas de Ansiedad, Motivación y Confianza y el Grado estudiado por los estudiantes, su género o el curso académico donde se encuentran. Sin embargo, las evidencias halladas no son significativas.

\section{CONCLUSIONES}

Como conclusiones a este trabajo destacaríamos en primer lugar la aceptación y valoración positiva recibida por parte de los estudiantes involucrados en las experiencias descritas anteriormente.

Los resultados obtenidos de la encuesta de ansiedad matemática (Tablas 6 y 7) nos llevan a concluir que las diferentes estrategias empleadas de forma continuada a lo largo de diferentes cursos tienen su reflejo en los niveles de ansiedad matemática que presentan los estudiantes. Por un lado, las medias de las tres subescalas de ansiedad muestran unos valores en ambas titulaciones por debajo del nivel neutral (3) en todos los cursos. Estos valores están por debajo de los obtenidos por otros trabajos que han analizado a estudiantes universitarios.

Por otro lado, en el caso del Grado en ADE, parece claro que aquellos estudiantes que han participado en más actividades (los de $1^{\circ}, 2^{\circ}$ y $3^{\circ}$ ) presentan niveles menores de ansiedad matemática que aquellos que no han estado involucrados en todas ellas (los alumnos de $4^{\circ}$ ).

Los estudiantes de IGE presentan menores niveles de ansiedad matemática en todos los cursos en comparación con los de ADE. Además, el nivel de ansiedad de los estudiantes de $4^{\circ}$ curso sigue disminuyendo. Una posible explicación a este resultado es el perfil diferencial de los estudiantes de IGE con respecto a los de ADE. Los resultados de $4^{\circ}$ curso de IGE presentan un valor medio muy bajo y también el menor valor de la desviación típica, que puede estar explicado por ser el curso con el menor número de respuestas a la encuesta y el perfil de los estudiantes.

De los ítems de ansiedad matemática, la subescala que mayor puntuación presenta es la de Ansiedad hacia los exámenes en ambas titulaciones y en los dos Grados.
Pretendemos seguir con la investigación de los resultados que miden la ansiedad matemática a lo largo de los próximos cursos, de forma que podamos obtener resultados más consistentes y valorar la evolución en el tiempo. Planteamos para futuras investigaciones analizar la relación entre estos resultados y el perfil de los estudiantes, así como profundizar en los resultados obtenidos en la subescala de ansiedad hacia los exámenes.

Es nuestro interés seguir profundizando en este camino que sin duda puede mejorar la preparación de nuestros estudiantes para un mundo en el que las matemáticas van a estar muy presentes.

\section{AGRADECIMIENTOS}

Queremos agradecer al Departamento de Calidad del Centro Universitario EDEM su colaboración indispensable en este proyecto.

\section{REFERENCIAS}

Acevedo, G. V., Arenas, T. Y. A., y Calderón, W. J. T. (2020). Relación entre ansiedad matemática y rendimiento académico en matemáticas en estudiantes de secundaria. Ciencias Psicológicas.

Auzmendi, E. (1992). Las actitudes hacia la matemáticaestadística en las enseñanzas medias y universitaria. Bilbao: Ediciones Mensajero.

Caraballo, A. M. M., Paralera, C., \& Tenorio, Á. F. (2017). Dificultades del alumnado en Económicas y Empresariales al enfrentarse al Cálculo Integral. Anales de ASEPUMA, 25, 5 .

Carrillo-Fernández, M., González-Concepción, C., y SosaMartín, D. (2013). Una triple visión sobre la adecuación de las Matemáticas impartidas en Bachillerato para los Grados en Economía y ADE. Anales de ASEPUMA, 21, 4-22.

Carrillo-Fernández, M., Cruz Báez, D. I., Gil Fariña, M. C., González Concepción, C., Pestano Gabino, C., y Sosa Martín, D. N. (2012). Evolución de los contenidos de Matemáticas en la PAU y relación con los conocimientos previos del alumnado en los Grados en Economía y ADE. Anales de XX Jornadas de ASEPUMA, Barcelona.

Cascón, J. M., García, M. D., Manrique, A., Santos, G., Muñoz, F. C., Bernalt, B. G., y Prieto, S. (2018). Valoración de un Curso Cero de Matemáticas para una Facultad de Economía y Empresa. Anales de ASEPUMA, 26, 3.

Castro, M. Á., García Ferrández, P. A., Sirvent Guijarro, A., Cabrera Sánchez, J., Bueno Vargas, A. M., Martín Alustiza, J. A., y Rodríguez, F. (2018). Conocimientos previos de matemáticas del alumnado en titulaciones de Ingeniería: un análisis sincrónico y diacrónico. El compromiso académico y social a través de la investigación e innovación educativas en la Enseñanza Superior. Rosabel Roig-Vila (Ed.). Cap 12.

Castro, M. Á., García Ferrández, P. A., Sirvent Guijarro, A., Martín Alustiza, J. A., y Rodríguez, F. (2019). Actitudes hacia las Matemáticas, conocimientos previos y resultados de evaluación: un estudio en primer curso de titulaciones de Ingeniería. Investigación e innovación en la Enseñanza Superior Nuevos contextos, nuevas ideas. Rosabel RoigVila (Ed.) Cap 6. 
Castro, M. Á., García Ferrández, P. A., Sirvent Guijarro, A., Reyes Perales, J. A., Martín Alustiza, J. A., y Rodríguez, F. (2020). Bagaje y actitudes: factores que ayudan a transitar con éxito a través de los primeros contenidos de Matemáticas en Ingeniería. La docencia en la Enseñanza Superior. Nuevas aportaciones desde la investigación e innovación educativas. Rosabel Roig-Vila (Ed.) Cap 8.

CE (Comisión Europea), (2017) Communication from the Commission to the European Parliament, the Council, the European Economic and Social Committee and the Committee of the Regions on a renewed EU agenda for higher education. Brussels, 30.5.2017 COM (2017) 247 final, 1-12.

CE (Comisión Europea) (2005) "Europe Needs More Scientists: Report by the High Level Group on Increasing Human Resources for Science and Technology".

De la Torre, L. D. P., y Palacín, J. G. A. (2019). Programa de nivelación académica y rendimiento académico de los estudiantes. Desafíos, 10(2), 100-5.

Delgado-Monge, I., Castro-Martínez, E., y Pérez-Tyteca, P. (2020). Estudio comparativo sobre ansiedad matemática entre estudiantes de Costa Rica y España. Revista Electrónica Educare, 24(2), 296-316.

Domènech-Casal, J. (2018). Aprendizaje Basado en Proyectos en el marco STEM: componentes didácticas para la Competencia Científica. Ápice. Revista de Educación Científica, 21(2), 29-42.

Drabekova, J., y Švecova, S. (2015). Solving the Microeconomics Problems by Using the Tools of GeoGebra. Proceedings ICABR, 235.

Fennema, E. y Sherman, J. (1976). Fennema-Sherman Mathematics Attitudes: Instruments Designed to Measure Attituted Toward the Learning of Mathematics by Males and Females. JSAS Catalog of Selected Documents in Psychology, 6, 31 (Ms. No. 1255). Journal for Research in Mathematics Education, 7, 324-326.

Foley, A. E., Herts, J. B., Borgonovi, F., Guerriero, S., Levine, S. C., y Beilock, S. L. (2017). The math anxietyperformance link: A global phenomenon. Current Directions in Psychological Science, 26(1), 52-58.

García, S. B., y Pineda, M. (2020). Aplicación de la Metodología de Rasch para la medición de los conocimientos matemáticos de los alumnos de ADE. Anales de ASEPUMA, 28, 2.

Gil Fariña, M. C., Carrillo-Fernández, M., Cruz Báez, D., González-Concepción, C., Pestano-Gabino, C., y Sosa Martin, D. (2014). Análisis de la formación matemática preuniversitaria en el periodo 2001-2012 y consecuencias en los estudios de Economía y Empresa: El caso de la Universidad de La Laguna. España. Revista Internacional de Estudios Organizacionales 3(1), 59-82.
Hohenwarter, M., et al. (2021). Geogebra. Obtenido de https://www.geogebra.org/

Majerek, D. (2014). Application of Geogebra for teaching mathematics. Advances in science and technology research journal, 8(24), 51-54.

Márquez Carmona, J. (2004). Una revisión de las evidencias de fiabilidad y validez de los cuestionarios de actitudes y ansiedad hacia la estadística. Statistics Education Research Journal, 3(1), 5-28.

Nortes Martínez-Artero, R. y Nortes Checa, A. (2017). Ansiedad, motivación y confianza hacia las Matemáticas en futuros maestros de Primaria. Números. Revista Didáctica de las Matemáticas, 95, 77-92

Luttenberger, S., Wimmer, S., y Paechter, M. (2018). Spotlight on math anxiety. Psychology research and behavior management, 11, 311 .

Paredes, Marlio (2018). El aprendizaje activo, el aprendizaje basado en proyectos y la educación STEM. Conferencia presentada en Ciclo de conferencias en Educación Matemática de Gemad (16 de junio de 2018). Bogotá.

Pérez, M. M. (2008). Aprendizaje basado en proyectos colaborativos. Una experiencia en educación superior. Laurus, 14(28), 158-180.

Pérez-Tyteca, P.; Castro Martínez, E.; Rico Romero, L.; Castro Martínez, E. (2011). Ansiedad matemática, género y ramas de conocimiento en alumnos universitarios. Enseñanza de las ciencias: revista de investigación y experiencias didácticas, 29(2), 237-50.

Picado-Alfaro, M. (2018). Sugerencias didácticas para la implementación de un curso de nivelación en matemáticas: La sistematización de una experiencia en la Universidad Nacional de Costa Rica. Revista electrónica educare, 22(3), 314-331.

Portalés, S., Estay, G., y Cabezas, M. (2017). Nivelación Académica En Matemática: ¿Un Factor Que Aporta A La Disminución Del Abandono?

Rodríguez-Muñiz, L. J., Crespo, R., Díaz, I., Fioravanti, M., García-Raffi, L. M., González-Vasco, M. I., ... y Mallavibarrena, R. (2020). Los estudios de matemáticas en el ámbito universitario. D. Martín De Diego, T. Chacón, G. Curbera, F. Marcellán, \& M. Siles (Coords.), Libro Blanco de las Matemáticas, 95-162. (Para matemáticas en los estudios de ADE.)

Rojas-Kramer, C. A., Escalera-Chávez, M. E., Moreno-García, E., y García-Santillán, A. (2017). Motivación, ansiedad, confianza, agrado y utilidad. Los factores que explican la actitud hacia las matemáticas en los estudiantes de Economía. Revista INFAD de Psicología. International Journal of Developmental and Educational Psychology, 2(1), 527-540. 